\title{
Precise expression of Fis1 is important for glucose responsiveness of beta cells
}

\author{
Julia Schultz', Rica Waterstradt', Tobias Kantowski', Annekatrin Rickmann', \\ Florian Reinhardt', Vladimir Sharoyko ${ }^{2}$, Hindrik Mulder ${ }^{2}$, Markus Tiedge ${ }^{1}$ \\ and Simone Baltrusch1 \\ 1Institute of Medical Biochemistry and Molecular Biology, University of Rostock, Rostock, Germany \\ 2Department of Clinical Sciences, Unit of Molecular Metabolism, Lund University Diabetes Centre, \\ Malmö, Sweden
}

Correspondence should be addressed to J Schultz or S Baltrusch Email

julia.schultz@med. uni-rostock.de or simone.baltrusch@med. uni-rostock.de

\begin{abstract}
Mitochondrial network functionality is vital for glucose-stimulated insulin secretion in pancreatic beta cells. Altered mitochondrial dynamics in pancreatic beta cells are thought to trigger the development of type 2 diabetes mellitus. Fission protein 1 (Fis1) might be a key player in this process. Thus, the aim of this study was to investigate mitochondrial morphology in dependence of beta cell function, after knockdown and overexpression of Fis 1 . We demonstrate that glucose-unresponsive cells with impaired glucose-stimulated insulin secretion (INS1-832/2) showed decreased mitochondrial dynamics compared with glucose-responsive cells (INS1-832/13). Accordingly, mitochondrial morphology visualised using MitoTracker staining differed between the two cell lines. INS1-832/2 cells formed elongated and clustered mitochondria, whereas INS1-832/13 cells showed a homogenous mitochondrial network. Fis 1 overexpression using lentiviral transduction significantly improved glucose-stimulated insulin secretion and mitochondrial network homogeneity in glucose-unresponsive cells. Conversely, Fis1 downregulation by shRNA, both in primary mouse beta cells and glucose-responsive INS1-832/13 cells, caused unresponsiveness and significantly greater numbers of elongated mitochondria. Overexpression of FIS1 in primary mouse beta cells indicated an upper limit at which higher FIS1 expression reduced glucose-stimulated insulin secretion. Thus, FIS1 was overexpressed stepwise up to a high concentration in RINm5F cells using the RheoSwitch system. Moderate FIS1 expression improved glucose-stimulated insulin secretion, whereas high expression resulted in loss of glucose responsiveness and in mitochondrial artificial loop structures and clustering. Our data confirm that FIS1 is a key regulator in pancreatic beta cells, because both glucosestimulated insulin secretion and mitochondrial dynamics were clearly adapted to precise expression levels of this fission protein.
\end{abstract}

\section{Key Words}

- pancreatic beta cells

- fission protein 1

- mitochondrial dynamics

- glucose-stimulated insulin secretion
(C) 2016 Society for Endocrinology Printed in Great Britain
Published by Bioscientifica Ltd
Journal of Endocrinology (2016) 230, 81-91 


\section{Introduction}

Mitochondria are highly dynamic organelles that form a tubular network in living cells. The mitochondrial network continuously undergoes fusion and fission events (Westermann 2008, Ishihara 2011), resulting in the sharing of proteins, solutes and metabolites between single mitochondria (Chen \& Chan 2005). This mechanism allows damaged mitochondria to recover their activity and maintain their metabolic function, whereas totally dysfunctional mitochondria are removed from the network by autophagy (Twig et al. 2008a,b). Mitochondrial fission-to-fusion imbalance results in fragmentation or elongation of mitochondria, and is associated with reduced cellular efficiency and integrity (Bereiter-Hahn \& Voth 1994, Smirnova et al. 2001, James et al. 2003, Yoon et al. 2003, Anello et al. 2005, Lee et al. 2007, Twig et al. 2008b, Huang et al. 2011). Mitochondrial dysfunctions have been implicated in several human pathologies (Liesa et al. 2009, Lu 2009, Van Laar \& Berman 2009) and are thought to contribute to the pathogenesis of type 2 diabetes mellitus (Lowell \& Shulman 2005, Civitarese \& Ravussin 2008, Zorzano et al. 2009).

Fusion of the mitochondrial membranes is coordinated by three proteins. The homologous mitofusins 1 and 2 (MFN1, MFN2) are important for outer membrane fusion, whereas the optic atrophy 1 (OPA1) protein affects inner membrane fusion (Westermann 2008, Chen \& Chan 2009). Mitochondrial fission is mediated by dynamin-related protein 1 (DRP1) and the small fission protein 1 (FIS1) (Smirnova et al. 2001, James et al. 2003, Yoon et al. 2003, Stojanovski et al. 2004, Westermann 2008, Chen \& Chan 2009). Maintenance of a functional mitochondrial network requires these nuclear encoded proteins to act in concert with each other and with cellular ionic and second messenger systems, a process that is still imperfectly understood (Hyde et al. 2010). It is assumed that FIS1 initiates mitochondrial fission via oligomerisation in the outer mitochondrial membrane and eventually triggers the mitochondrial life cycle (Serasinghe \& Yoon 2008, Stiles \& Shirihai 2012). In Saccharomyces cerevisiae yeast, complex formation of Fis1 and Drp1 on the outer mitochondrial membrane is mediated by Mdv1, a protein that is not expressed in mammals (Stojanovski et al. 2004, Wells et al. 2007, Zhang \& Chan 2007). Human FIS1 appears not to be a direct anchor for DRP1, contrary to earlier opinions (Yoon et al. 2003, Otera et al. 2010). However, mitochondrial fission factor (MFF) (Otera et al. 2010) and the mitochondrial dynamics proteins (MiD49/ MiD51) have recently been identified (Palmer et al. 2011,
Palmer et al. 2013): these may mediate recruitment of DRP1 to the outer mitochondrial membrane in eukaryotic cells (Loson et al. 2013). Unlike FIS1, DRP1 is a soluble GTPase that can interact with and separate membranes, and is thus able to finalise mitochondrial fission (Zhu et al. 2004).

Both mitochondrial fission-to-fusion imbalance and a reduced overall level of mitochondrial dynamics have been linked with decreased glucose-stimulated insulin secretion in pancreatic beta cells (Deng et al. 2004, Anello et al. 2005). Excess or lack of one or more fusion and fission protein in beta cells causes mitochondrial fragmentation, elongation or clustering (Maechler \& Wollheim 2000, Stojanovski et al. 2004, Yu et al. 2005, Lee et al. 2007, Park et al. 2008, Yoon et al. 2011). The essential contribution of the ubiquitously expressed FIS1 as a trigger of mitochondrial dynamics is generally well accepted (Stojanovski et al. 2004, Mouli et al. 2009), but recently, it has been argued that other factors might play a more important role (Otera et al. 2010, Palmer et al. 2013). Overexpression and downregulation of FIS1 in beta cells has yielded puzzling results (Park et al. 2008, Twig et al. 2008a, Molina et al. 2009). In two studies, reduction in FIS1 expression in INS1 cells using RNA interference decreased maximal respiratory capacity and glucose-stimulated insulin secretion (Twig et al. 2008a, Molina et al. 2009), with only minimal effects on mitochondrial morphology. However, expression levels of other fusion and fission proteins were not investigated. Elsewhere, overexpression of FIS1 in INS1E cells caused not only mitochondrial fragmentation but also mitochondrial aggregation (Park et al. 2008). INS1E cells with high FIS1 expression showed a reduced response to glucose stimulus with only minor increases in ATP production, mitochondrial membrane potential and $\mathrm{Ca}^{2+}$ concentration and, in consequence, impaired glucose-stimulated insulin secretion (Park et al. 2008). The aim of the present study was, therefore, to elucidate the effects of downregulation and overexpression of FIS1 on mitochondrial morphology and glucose-stimulated insulin secretion in different beta cell lines and primary mouse beta cells.

\section{Materials and methods}

\section{Lentivirus generation}

Lentiviruses were generated using the Trans Lentiviral Packaging Kit (Thermo Fisher Scientific) and the HEK293T producer cell line according to the manufacturer's instructions. For overexpression, human FIS1 was

Published by Bioscientifica Ltd. 
subcloned from the pOtb7/hFis1 vector (Thermo Fisher Scientific) in the EcoRI and SmaI sites of the pLVX-Puro vector. shRNA for Fis1 in the pLKO.1 vector (Thermo Fisher Scientific) was used for downregulation. Empty vector served as control during viral transduction.

\section{Cell culture}

Selection of clonal rat glucose-responsive INS1-832/13 and glucose-unresponsive INS1-832/2 cells was performed as described previously (Hohmeier et al. 2000, Malmgren et al. 2009). INS1 cells were transduced with lentiviruses for 3 days and cell clones were selected through resistance against puromycin $(500 \mu \mathrm{g} / \mathrm{mL})$. Generation of insulinproducing RINm5F-R cells containing the RheoSwitch Mammalian Inducible Expression System (New England BioLabs; Beverly, MA, USA) has been reported previously (Schmitt et al. 2011). The cDNA of human FIS1 fused to the yellow fluorescence protein (EYFP) was subcloned in the BamHI and SalI sites of the pNEBR-X1 vector (New England BioLabs, Beverly, MA, USA). Stable RINm5FR-FIS1 cell clones were selected after transfection with the pNEBR-X1-Fis1 vector through resistance against hygromycin $(250 \mu \mathrm{g} / \mathrm{mL})$ and puromycin $(100 \mu \mathrm{g} / \mathrm{mL})$. Fis1 expression was induced by incubation with 100 or $500 \mathrm{nmol} / \mathrm{L}$ of the RSL1 ligand for $24 \mathrm{~h}$. RINm5F-R and INS1 cells were cultured in RPMI 1640 media containing $10 \mathrm{mmol} / \mathrm{L}$ glucose and supplemented with $10 \%$ foetal bovine serum and $5 \%$ penicillin/streptomycin, $1 \mathrm{mmol} / \mathrm{L}$ sodium pyruvate, $10 \mathrm{mmol} / \mathrm{L}$ HEPES, $50 \mu \mathrm{M}$ 2-mercaptoethanol and $200 \mathrm{mmol} / \mathrm{L}$ glutamine in a humidified atmosphere at $37^{\circ} \mathrm{C}$ and $5 \% \mathrm{CO}_{2}$.

\section{Mouse pancreatic islets}

Mouse pancreatic islets were isolated from male 12-weekold C57BL/6 mice (Jackson Laboratory) by collagenase P digestion (Roche Diagnostics) and Ficoll gradient purification (Ficoll PM 400; Sigma). Islets were dissociated using calcium-free medium, seeded on collagen-coated well plates or PCA-coated Lab-Tek chamber slides (Nunc, Naperville, IL, USA) for microscopy and transduced with lentiviruses for 3 days.

\section{Real-time PCR}

Total RNA samples were prepared using the RNeasy total RNA isolation kit (Qiagen) and quantified using a spectrophotometer (ND-2000, PeqLab, Erlangen,
Germany). RNA samples were reverse-transcribed into cDNA using the Maxima First Strand cDNA Synthesis Kit (Fermentas, St Leon-Rot, Germany). Gene expression was quantified using TaqMan Universal PCR Master Mix and the following assays: Rn_01480914_m1 for Fis1, Rn_01405085 for Drp1, Rn_01404701_m1 for OPA1, Rn_01462175_m1 for Mfn1, Rn_01639201_m1 for Mfn2, Rn_00580051_m1 for the mitochondrial transcription factor TFAM and Rn_00821325_g1 for the voltagedependent anion channel (Applied Biosystems). PCR reactions were performed in triplicates using the 7900HT Real-Time PCR System (Applied Biosystems). Relative expression levels were calculated with the comparative $\left(2^{-\Delta \Delta \mathrm{Ct})}\right.$ method. Gene expression levels were normalised to Gapdh (4352338E-0908012).

\section{Western blot analyses}

Cells were extracted with RIPA buffer $(50 \mathrm{mmol} / \mathrm{L}$ Tris-HCl $\mathrm{pH} 7.4,150 \mathrm{mmol} / \mathrm{L} \mathrm{NaCl}, 1 \%$ Triton X-100, 1\% sodium deoxycholate, $0.1 \%$ SDS, $1 \mathrm{mmol} / \mathrm{L}$ EDTA, protease inhibitors) and centrifuged for $10 \mathrm{~min}$ at $10,000 \times \boldsymbol{g}$. Forty micrograms of proteins were separated by SDS-PAGE and blotted onto Roti Fluoro PVDF membrane (Roth, Karlsruhe, Germany). The membranes were probed for $1 \mathrm{~h}$ at room temperature with the following primary antibodies: anti-beta-tubulin (1:1000), anti-FIS1 (1:1000), anti-GAPDH (1:1000) (Santa-Cruz Biotechnology) and the MitoProfile Total OXPHOS Rodent WB Antibody Cocktail (MitoSciences, Eugene, OR, USA). Immunoreactive bands were visualised using the following fluorescence-labelled secondary antibodies: IRDye 680, IRDye $800 \mathrm{CW}$ and analysed via the Odyssey imaging system. Densitometry measurements of bands were performed using the Odyssey infrared imaging system (LI-COR, Lincoln, NE, USA) and normalised to beta-tubulin or GAPDH expression.

\section{Immunohistochemistry}

Cells were fixed with $4 \%$ formaldehyde for $15 \mathrm{~min}$ and permeabilised with $0.2 \%$ Tween 20 for $5 \mathrm{~min}$ in phosphatebuffered saline. Finally, cells were stained for $1 \mathrm{~h}$ with the following primary antibodies: anti-FIS1 (1:100) (SantaCruz Biotechnology) and anti-insulin (1:100) (Abcam). Positive signals were visualised using Cy5 or FITCcoupled secondary antibodies (1:250) (Molecular Probes Invitrogen). The samples were counterstained with DAPI and mounted using VECTASHIELD mounting medium (Vector Laboratories, Peterborough, UK). Cells were 


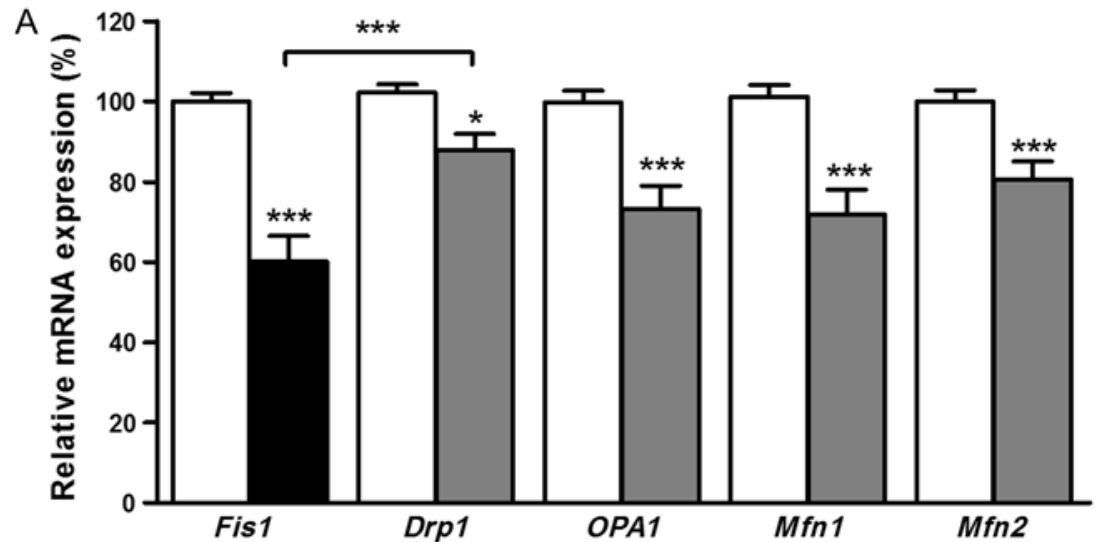

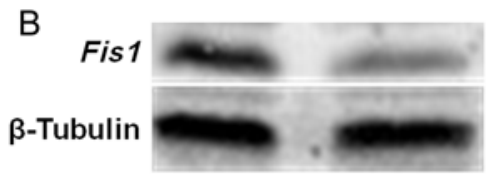

C

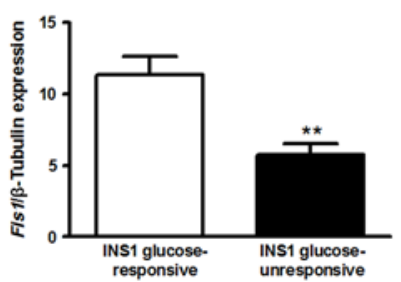

analysed using a Fluoview FV10i confocal microscope (Olympus). A minimum of 10 sections per slide from three independent experiments were analysed using Fluoview-SW (Olympus) software.

\section{Glucose-stimulated insulin secretion}

INS1 and RINm5F-R-Fis1 cells and mouse beta cells were incubated for $1 \mathrm{~h}$ in bicarbonate-buffered Krebs-Ringer solution without glucose, supplemented with $0.1 \%$ albumin. Subsequently, cells were incubated for $1 \mathrm{~h}$ in Krebs-Ringer solution containing 3, 10, 20 and $25 \mathrm{mmol} / \mathrm{L}$ glucose, respectively. Finally, $1 \mathrm{~mL}$ of the incubation buffer from each well was collected and gently centrifuged to remove detached cells. The secreted insulin was measured in the final supernatant by ELISA (Mercodia, Uppsala, Sweden). The cells were scraped off and homogenised by sonication in Krebs-Ringer solution. In soluble fractions, insulin content was measured by ELISA and protein content by Bradford protein assay.

\section{Mitochondrial morphology}

Glucose-responsive and glucose-unresponsive INS1 and RIN5mF-R-Fis1 cells seeded on glass-bottom dishes

\section{Figure 1}

Reduced expression of key proteins of mitochondrial dynamics in INS1 glucoseunresponsive compared with glucose-responsive cells. (A) Gene expression of Fis1, Drp1, Opa1, $M f n 1$ and Mfn2 in INS1 glucose-responsive (white bars) and glucose-unresponsive (Fis1, black bar; other proteins: grey bars) cells normalised to Gapdh expression. Data are presented as mean \pm S.E.M. from six independent experiments; ${ }^{*} P<0.05, * * * P<0.001$ (ANOVA/Bonferroni's test). FIS1 protein expression in INS1 glucose-responsive and glucose-unresponsive cells was analysed by western blotting $(B, C)$ and immunofluorescence (D). Scale bar: $10 \mu \mathrm{m}$. (C) Data are presented as mean \pm S.E.M. from four independent experiments; $* * P<0.01$ (Student's $t$-test).

(MatTak Corporation, Ashland, MA, USA) were stained with $20 \mathrm{nmol} / \mathrm{L}$ MitoTracker Green FM (Molecular Probes Invitrogen) for $30 \mathrm{~min}$ at $37^{\circ} \mathrm{C}$. Primary mouse beta cells were fixed with $4 \%$ paraformaldehyde and stained with $20 \mathrm{nmol} / \mathrm{L}$ MitoTracker Deep Red FM (Molecular Probes Invitrogen) for $30 \mathrm{~min}$ at $37^{\circ} \mathrm{C}$. Finally, mitochondrial morphology was visualised using a Fluoview FV10i (Olympus) microscope. Mitochondrial size was quantified using AutoQuant X2 software (Media Cybernetics, Bethesda, MD, USA). The same fluorescence threshold

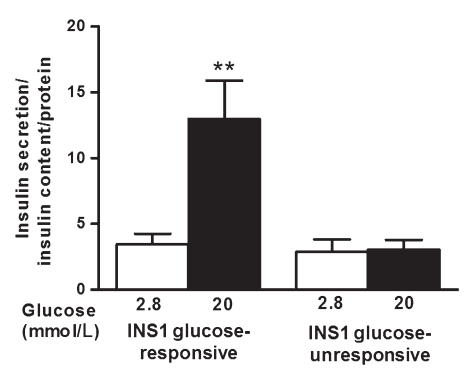

Figure 2

Reduced glucose-stimulated insulin secretion in INS1 glucoseunresponsive compared with glucose-responsive cells. Basal (white bars) and stimulated (black bars) insulin secretions are expressed as mean \pm S.E.M. from six independent experiments; $* * P<0.01$ (ANOVA/ Bonferroni's test). 
to detect single objects (mitochondria) per cell was used for all images. Each automatically detected object was numbered by the software algorithm and finally calculated with respect to size.

\section{Statistical analysis}

Data are expressed as mean \pm s.e.M. Statistical analyses were performed by ANOVA followed by Bonferroni's test or Student's $t$-test using the Prism analysis program (GraphPad). $P<0.05$ was considered to be statistically significant.
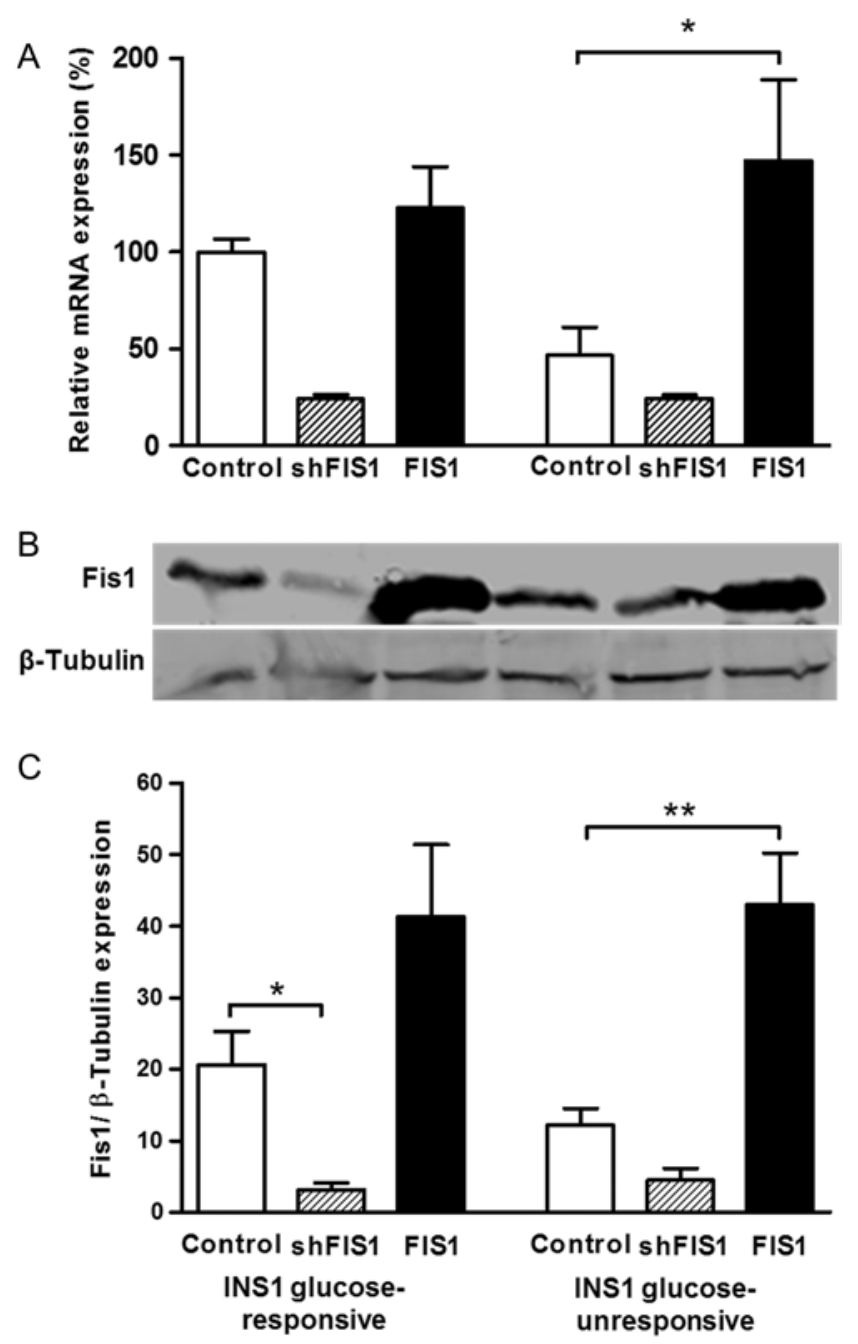

Figure 3

Downregulation and overexpression of FIS1 in INS1 glucose-responsive and glucose-unresponsive cells. Fis1 gene (A) and protein (B and C) expression in INS1 cells after transduction with control (white bars), shFis1 (striped bars) or Fis1 (black bars) virus. Data are presented as mean \pm s.E.M. from six (A) or three (C) independent experiments; ${ }^{*} P<0.05$, $* * P<0.01$ (ANOVA/Bonferroni's test).

\section{Results}

Reduced expression of proteins regulating mitochondrial dynamics in INS1 glucose-unresponsive cells compared with glucose-responsive cells

mRNA expression levels of Fis1, Drp1, Opa1, Mfn1 and $M f n 2$ were investigated in INS1 glucose-responsive and glucose-unresponsive cells. Gene expression of these key regulators of mitochondrial fusion and fission was significantly reduced in INS1 glucose-unresponsive cells compared with glucose-responsive cells by $20-40 \%$ (Fig. 1A). The greatest reduction was observed for Fis1,
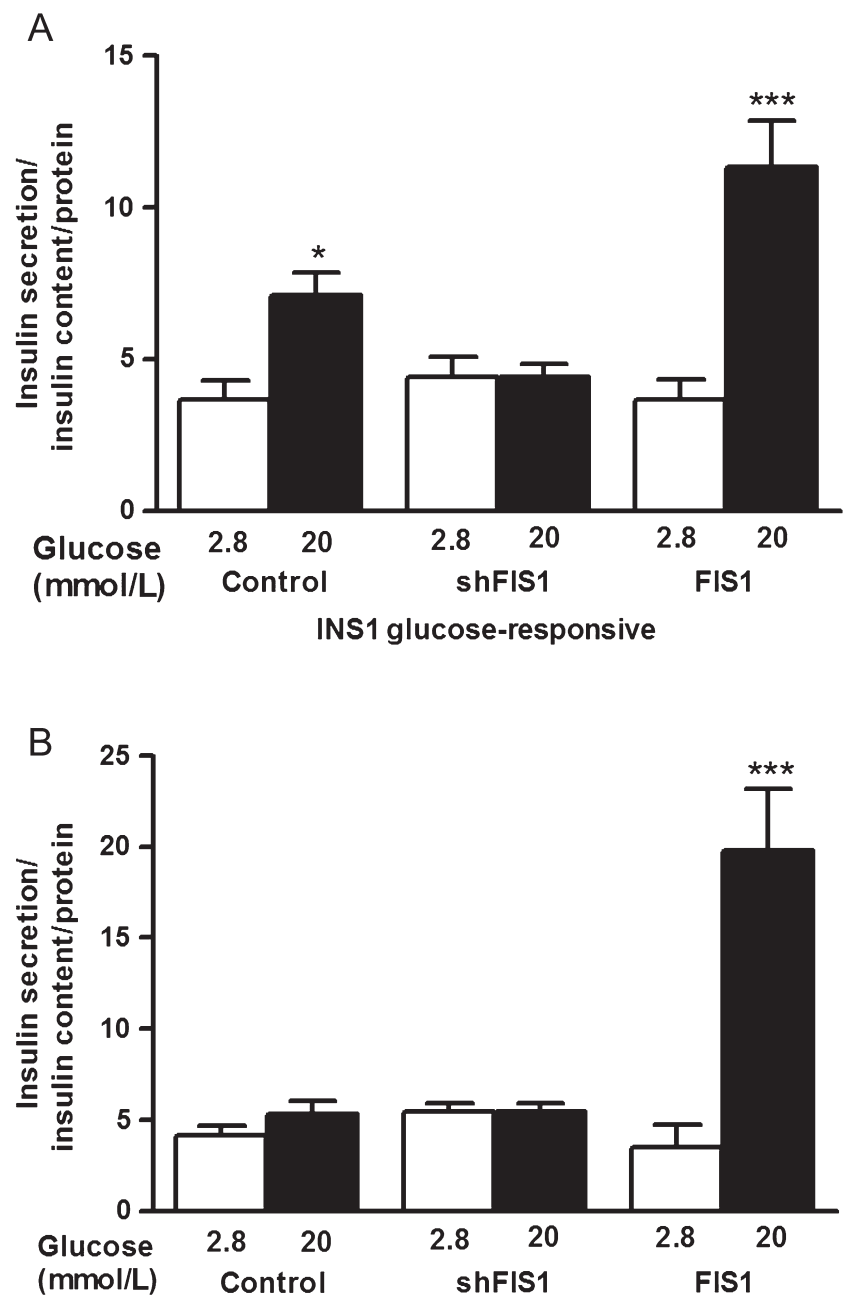

INS1 glucose-unresponsive

Figure 4

Effects of downregulation and overexpression of FIS1 on glucosestimulated insulin secretion in INS1 glucose-responsive and glucoseunresponsive cells. Basal (white bars) and stimulated (black bars) insulin secretion in INS1 glucose-responsive (A) and glucose-unresponsive cells (B) after transduction with control, shFis1 or Fis1 virus. Data are presented as mean \pm S.E.M. from six independent experiments; ${ }^{*} P<0.05$, $* * * P<0.001$ (ANOVA/Bonferroni's test).

Published by Bioscientifica Ltd. 
as confirmed at the protein level by western blotting (Fig. 1B) and immunofluorescence analyses (Fig. 1C). Measurements of basal and glucose-stimulated insulin secretion confirmed the unresponsiveness of INS1 832/2 glucose-unresponsive cells (Fig. 2). By contrast, INS1 832/13 glucose-responsive cells showed a significant 3.7-fold increase in insulin secretion after stimulation with $20 \mathrm{mmol} / \mathrm{L}$ glucose (compared with $2.8 \mathrm{mmol} / \mathrm{L}$ ) (Fig. 2).

\section{Correlation of Fis 1 expression level with glucose- stimulated insulin secretion in INS1 cells}

To evaluate the effect of Fis1 expression on insulin secretion, stable transduction of both INS1 cell lines was performed with viruses containing Fis1 to induce overexpression or with shFis1 for downregulation. Virus transduction without insert served as control. Changes in Fis1 expression were confirmed at the gene and protein level (Fig. 3). Basal insulin secretion remained unaffected by the FIS1 expression level in both INS1 cell lines (Fig. 4). In INS1 glucose-responsive cells stimulated insulin secretion was reduced by $47 \%$ after FIS1 downregulation and increased by 59\% after FIS1 overexpression (Fig. 4A). In INS1 cells already glucose-unresponsive, FIS1 downregulation had no impact on stimulated insulin secretion, but FIS1 overexpression resulted in an increase of $268 \%$ (Fig. 4B). Significant glucose responsiveness, therefore, correlated with a high FIS1 expression level in INS1 cells (Fig. 4).
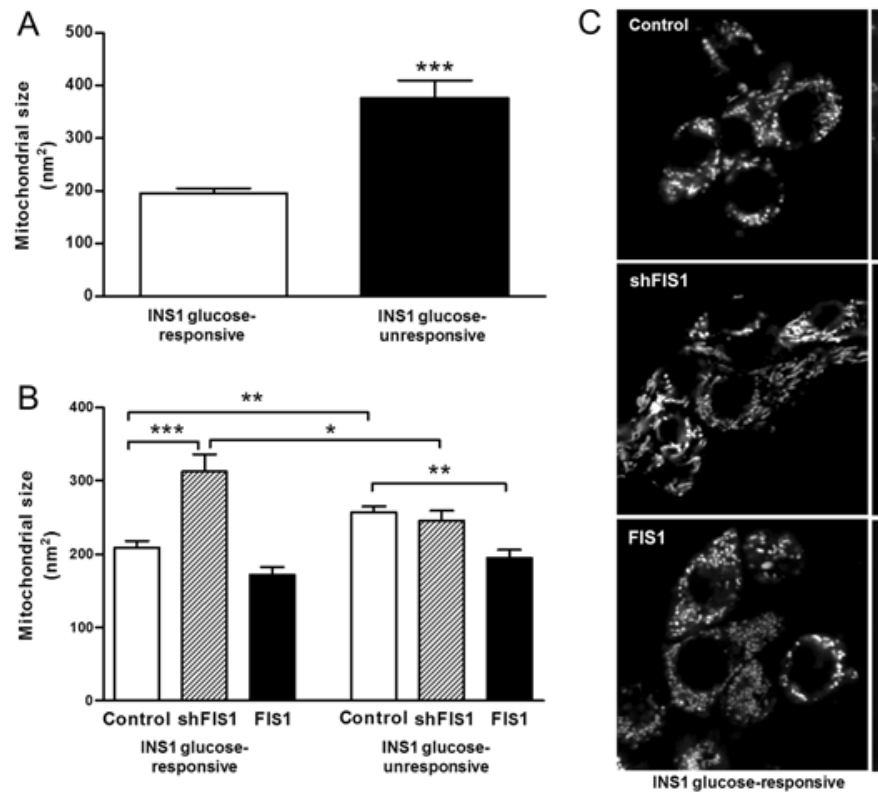

http://joe.endocrinology-journals.org DOI: 10.1530/JOE-16-0111

(c) 2016 Society for Endocrinology Printed in Great Britain

\section{Mitochondrial morphology, function and regulation relative to FIS1 expression}

The mitochondrial network structure in INS1 cells was visualised in living cells by staining with MitoTracker Green. INS1 glucose-responsive cells showed homogenous distribution of mitochondria, whereas elongated mitochondria were clearly evident in glucose-unresponsive cells. This observation was expressed quantitatively by automated detection of each single mitochondrion and determining its size. Mitochondrial size in INS1 glucoseunresponsive cells was significantly larger than in glucose-responsive cells (Fig. 5A). Downregulation of FIS1 in INS1 glucose-responsive cells resulted in a significant $50 \%$ increase in mitochondrial size, compared with an $18 \%$ reduction following overexpression (Fig. 5B). In INS1 glucose-unresponsive cells, down-regulation of FIS1 had no impact on mitochondrial size, whereas overexpression resulted in a significant $24 \%$ reduction (Fig. 5B). Finally, overexpression of FIS1 at the same level (Fig. 3C) was associated with a similar mitochondrial size in glucoseresponsive $\left(172 \pm 11 \mathrm{~nm}^{2}\right)$ and glucose-unresponsive $\left(195 \pm 11 \mathrm{~nm}^{2}\right)$ cells (Fig. 5B). Despite comparable low FIS1 expression levels in both cells lines after downregulation (Fig. 3C), mitochondrial size was significantly smaller in glucose-unresponsive $\left(245 \pm 13 \mathrm{~nm}^{2}\right)$ compared with glucose-responsive $\left(312 \pm 24 \mathrm{~nm}^{2}\right)$ cells.

The two INS1 cell lines showed different expression levels of the single complexes of oxidative phosphorylation and ATP synthase, which are mandatory elements of mitochondrial energy production (Malmgren et al. 2009).

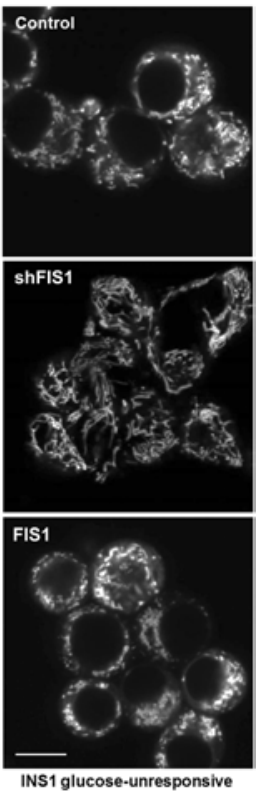

Figure 5

Effects of downregulation and overexpression of FIS1 on mitochondrial morphology in INS1 glucose-responsive and glucose-unresponsive cells. (A) Mitochondrial size was calculated in MitoTracker Green-stained glucose-responsive (white bar) and glucose-unresponsive (black bar) INS-1 cells, and in INS1 cells after transduction ( $B$ and $C$ ) with control (white bars), shFis1 (striped bars) or Fis1 (black bars) virus. (A, B) Data are presented as the mean \pm S.E.M. from four independent experiments; $(A) * * * P<0.001$ (Student's $t$-test), $(B) * P<0.05, * * P<0.01$, $* * * P<0.001$ (ANOVA/Bonferroni's test). (C) Representative cells with mitochondria highlighted by MitoTracker Green. Scale bar: $10 \mu \mathrm{m}$. 
A

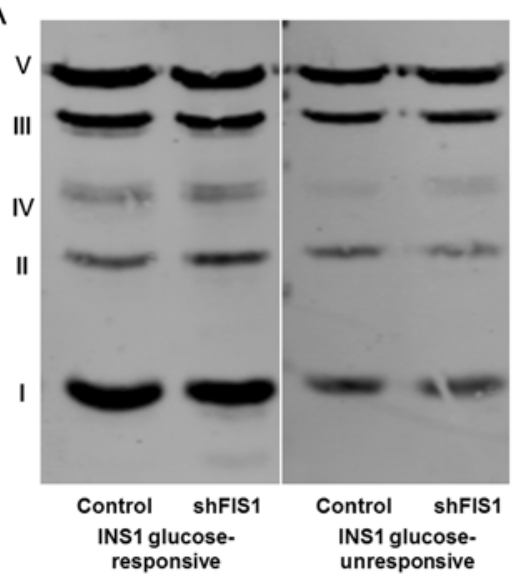

B
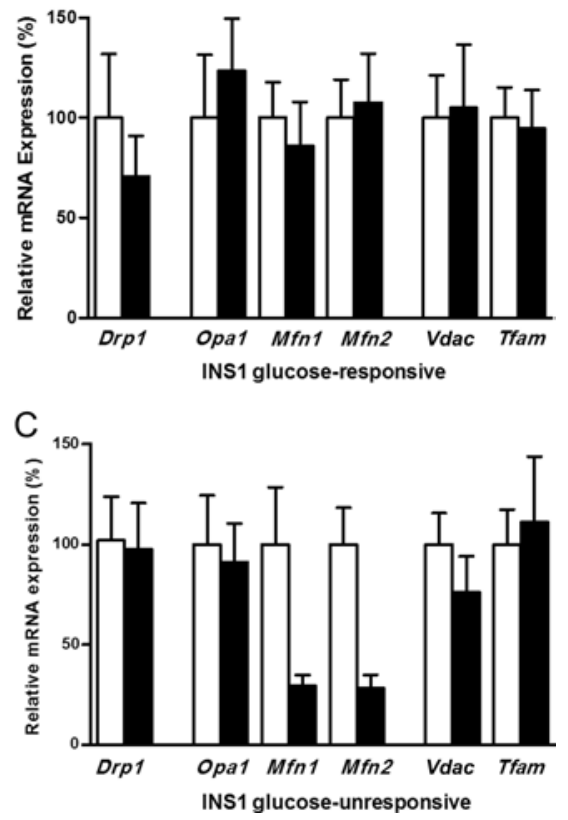

Figure 6

Effects of downregulation on gene and protein expression related to mitochondrial function in INS1 glucose-responsive and glucose-unresponsive cells. (A) Representative western blot assay of the five complexes of oxidative phosphorylation from three independent experiments. Gene expression of Drp1, Opa1, Mfn1, Mfn2, voltage-dependent anion channel and the mitochondrial transcription factor TFAM in glucose-responsive (B) and glucose-unresponsive (C) INS1 cells after transduction with control (white bars) or shFis1 (black bars) virus. Data are presented as the mean \pm s.E.M. from six independent experiments.
This protein expression pattern remained unaffected by FIS1 downregulation (Fig. 6A). Also, gene expressions of the voltage-dependent anion channel, the most highly expressed mitochondrial protein, and of TFAM, the mitochondrial transcription factor, as well as of $D R P 1$ and OPA1 were unchanged by FIS1 downregulation in glucoseresponsive (Fig. 6B) and glucose-unresponsive (Fig. 6C) cells. $M f n 1$ and $M f n 2$ gene expression was reduced in INS1 glucose-unresponsive but not in glucose-responsive cells (Fig. 6B and C).

\section{Downregulation and overexpression of FIS1 affects glucose-stimulated insulin secretion and mitochondrial morphology in primary mouse beta cells}

The mitochondrial network structure in dispersed primary mouse islets was visualised after fixation by staining with
MitoTracker Deep Red. Insulin-positive beta cells infected with control virus showed a homogenous mitochondrial network with only few fragmented or elongated mitochondria (Fig. 7A). Overexpression of FIS1 evoked a high mitochondrial elongation level in beta cells (Fig. 7A). Downregulation of FIS1 changed the mitochondrial network structure to clusters (Fig. 7A). Control cells showed a significant 3.6-fold increase in insulin secretion after stimulation with $20 \mathrm{mmol} / \mathrm{L}$ glucose compared with $2.8 \mathrm{mmol} / \mathrm{L}$ glucose (Fig. 7B). Both, downregulation and overexpression of FIS1 resulted in only a 1.8-fold increase, i.e. reduced glucose responsiveness (Fig. 7B). FIS1 downregulation resulted in a significant reduction in both basal and stimulated insulin secretion compared with controls (Fig. 7B). Thus, physiological (but not supraphysiological) FIS1 expression was best for appropriate glucose responsiveness in beta cells (Fig. 7).
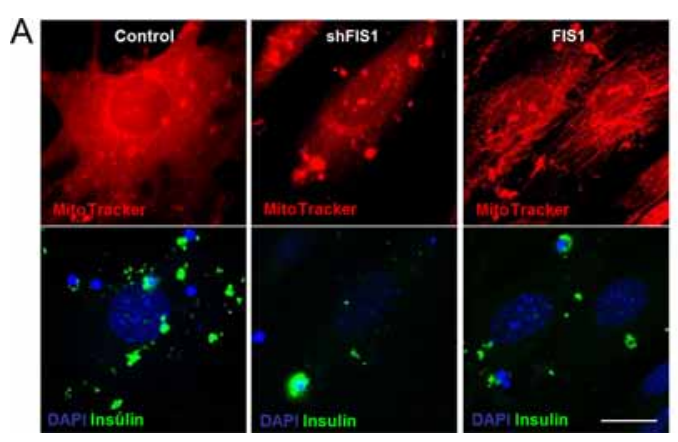

B

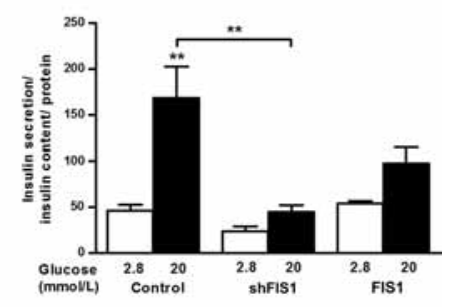

Figure 7

Effects of downregulation and overexpression of FIS1 on mitochondrial morphology and glucose-stimulated insulin secretion in primary mouse beta cells. (A) Mitochondrial morphology and (B) basal (white bars) and stimulated (black bars) insulin secretion in dispersed mouse islets after transduction with control, shFis1 or Fis1 virus. (B) Data are expressed as the mean \pm S.E.M. from three independent experiments; $* * P<0.01$ (ANOVA/Bonferroni's test). (A) Representative cells with MitoTracker Deep Red and DAPI staining, and anti-insulin immunofluorescence. Scale bar: $10 \mu \mathrm{m}$. http://joe.endocrinology-journals.org DOI: $10.1530 / J O E-16-0111$
(C) 2016 Society for Endocrinology Printed in Great Britain 
A
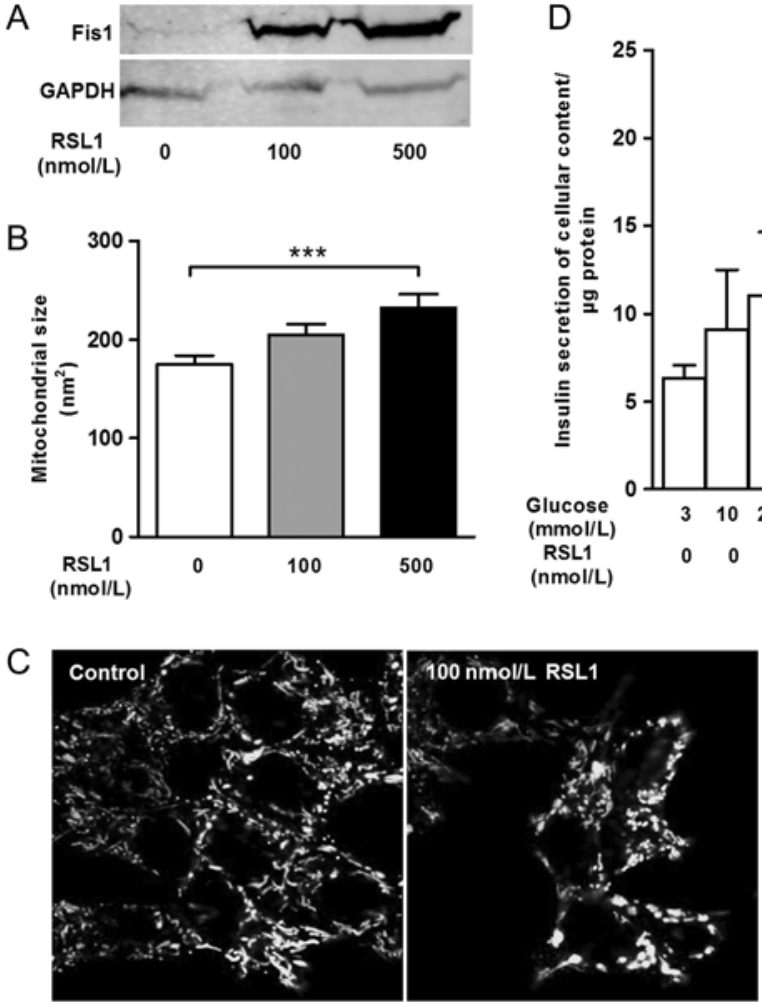
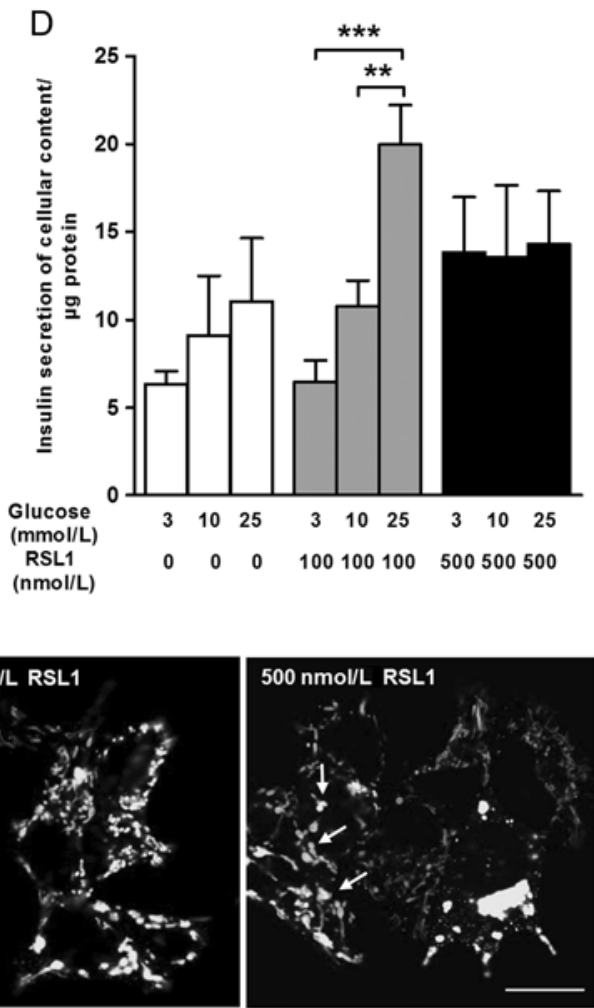

Figure 8

Stepwise overexpression of FIS1 in RINm5F-REYFP-Fis 1 cells. RINm5F-R-EYFP-FIS1 cells were pre-incubated for $48 \mathrm{~h}$ in the absence (white bars) or presence of 100 (grey bars) or $500 \mathrm{nmol} / \mathrm{L}$ (black bars) of the inductor RSL1.

(A) Representative western blot assay of FIS1 and GAPDH from three independent experiments. Mitochondrial morphology was determined after MitoTracker Green staining. Mitochondrial size (B) is presented as the mean \pm s.E.M. from three independent experiments; $* * * P<0.001$ (ANOVA/ Bonferroni's test) and representative cells (C) are shown. Examples of loop-shaped mitochondria are marked by white arrows. Scale bar: $10 \mu \mathrm{m}$. (D) Basal (3 $\mathrm{mmol} / \mathrm{L}$ glucose) and stimulated (10 and $25 \mathrm{mmol} / \mathrm{L}$ glucose) insulin secretion was determined. Data are presented as mean \pm S.E.M. from six independent experiments; $* * P<0.01$ $* * * P<0.001$ (ANOVA/Bonferroni's test).
Positive effect of FIS1 overexpression on glucosestimulated insulin secretion is limited in RINm5F cells

RINm5F cells are endowed with a very low FIS1 expression level compared with INS1 glucose-responsive cells (Figs 1B and 8A). FIS1 expression was increased stepwise using the RheoSwitch system (Schmitt et al. 2011). Moderate (100 nmol/L RSL1) and high (500 nmol/L RSL1) expression of FIS1 (Fig. 8A) resulted in an increase in mitochondrial size in RINm5F-R-EYFP-Fis1 cells (Fig. 8B). While only a few mitochondrial aggregates were present following moderate FIS1 expression, high FIS1 expression resulted in mitochondrial clustering and formation of loop-shaped mitochondria in RINm5F-R-EYFP-FIS1 cells (Fig. 8C, white arrows). Glucose-stimulated insulin secretion in RINm5FR-EYFP-FIS1 cells was significantly improved by moderate FIS1 expression, but was totally disrupted by high FIS1 expression (Fig. 8D).

\section{Discussion}

In pancreatic beta cells, mitochondria form an interface between nutrient input and insulin secretion. The organelles move in a highly dynamic tubular network that is apparently adapted to the demands of stimulussecretion coupling (Kuznetsov et al. 2010). Various data suggest that any changes in mitochondrial dynamics can lead to beta cell dysregulation that is linked with functional abnormalities (Maechler \& Wollheim 2000, Wollheim 2000, Detmer \& Chan 2007, Yoon et al. 2011). Mitochondrial fusion has been described as a brief phase, which is followed by the accumulation of FIS1 to the outer mitochondrial membrane and initiation of fission. Accordingly, downregulation of FIS1 in eukaryotic cells results in abnormally elongated mitochondrial tubules (Yoon et al. 2003, Lee et al. 2007). Conversely, Park and coworkers have reported a high mitochondrial fragmentation rate in human pancreatic islet cells after infection with adenoviruses encoding for human FIS1 (Park et al. 2008).

Hohmeier and coworkers isolated individual clones from rat insulin-producing INS1 cells and established glucose-responsive and glucose-unresponsive subpopulations (Hohmeier et al. 2000). Further investigations revealed that tight coupling between glucose and mitochondrial metabolism is important for robust stimulus-secretion coupling in INS1-832/13 cells and is impaired in the glucose-unresponsive INS1-832/2 clone (Malmgren et al. 2009). In the present study, we compared the regulation of mitochondrial morphology in both subpopulations. Gene expression of all proteins involved in fusion and fission was 
significantly reduced in INS1 glucose-unresponsive compared with glucose-responsive cells, indicating a diminished mitochondrial life cycle. Analyses of the mitochondrial network structure in living INS1 cells showed greater elongation in glucose-unresponsive cells. This observation was consistent with the most pronounced reduction in FIS1 from all fusion and fission proteins in glucose-unresponsive versus glucose-responsive cells.

To test the hypothesis that FIS1 expression may be linked to glucose responsiveness in INS1 cells, we established downregulation and overexpression of the protein by lentiviral transduction. Overexpression of FIS1 in glucose-unresponsive cells actually increased the response to glucose, enhanced the insulin secretion and induced a more homogenous mitochondrial network. Conversely, downregulation of FIS1 in glucose-responsive cells diminished glucose-stimulated insulin secretion and increased mitochondrial elongation. It was noteworthy that neither expression of oxidative phosphorylation complexes and ATP synthase nor mitochondrial mass was changed by downregulation of FIS1. Thus, our study suggests that insulin secretion in INS1 cells is reliant on FIS1 expression. This hypothesis was verified in primary mouse beta cells, where downregulation of FIS1 was accompanied by diminished glucose-stimulated insulin secretion and an inhomogenous mitochondrial network.

However, after overexpression of FIS1, we also observed a reduction in glucose-stimulated insulin secretion and some mitochondrial clustering in primary mouse beta cells. This accords with findings from a previous study in which INS1E cells overexpressing FIS1 showed reduced mitochondrial length and impaired glucose-stimulated insulin secretion (Park et al. 2008). By contrast, in glucoseresponsive INS1 cells, a further improvement in glucose responsiveness was elicited by FIS1 overexpression. Because insulin-secreting cell lines differ between themselves and by comparison with primary beta cells in terms of their metabolomics, this argues in favour of feedback mechanisms that adjust FIS1 expression to the current need for mitochondrial network regulation in a given cell population. Adaptation of mitochondrial dynamics may rely on the mutual regulation of fusion and fission genes (Stiles \& Shirihai 2012). Moreover, the influence of cell morphology and formation of cell-cell contacts on glucose responsiveness and mitochondrial network appearance should be considered as an independent effect. INS1 cells grow as a monolayer with a somewhat higher degree of clustering in the glucose-responsive compared with the glucose-unresponsive subpopulation, but this differs from the in vivo situation where beta cells grow in an islet architecture.

In RINm5F cells, another rat insulin-producing cell line with limited glucose responsiveness, we observed a low FIS1 expression level comparable with that of glucoseunresponsive cells, but a less homogenous mitochondrial network with a smaller mean mitochondrial area. An inducible expression system established in a previous investigation (Schmitt et al. 2011) was used to increase FIS1 stepwise in RINm5F-R-EYFP-Fis1 cells: while glucose-stimulated insulin secretion was improved by balanced FIS1 overexpression, it was diminished by high overexpression. These data suggest an upper limit of FIS1 expression at which insulin-secreting cells become compromised. Above that level, clustering of mitochondria was also observed, culminating in huge aggregates at high FIS1 expression levels. According to Frieden and co-workers, overexpression of FIS1 in HeLa cells induces rapid and complete fragmentation of mitochondria, leading to perinuclear clustering (Frieden et al. 2004). Other studies have demonstrated the formation of aggregated mitochondria after overexpression of the fusion protein MFN2 as a terminal stage of hyper-fusion (Huang 2006, Santel \& Fuller 2001). Regardless of their origin, mitochondrial clusters seem to sustainably affect cell function because they cannot be removed by mitochondrial autophagy (Mouli et al. 2009). Moreover, there is evidence that mitochondrial clustering triggers the development of type 2 diabetes mellitus (Dlaskova et al. 2010). Frequent occurrence of loop-shaped mitochondria was also observed after FIS1 expression. Such artificial structures appear to be separated from the mitochondrial network and have been described in response to metabolic stress (Liu \& Hajnoczky 2011). The role of FIS1 in primary beta cells, therefore, needs to be investigated further using knockout techniques in animal models of type 2 diabetes mellitus.

In summary, we have provided evidence to support the argument that FIS1 is a key regulator in pancreatic beta cells. Our data suggest that appropriate glucosestimulated insulin secretion is dependent on a precise FIS1 expression level that adapts mitochondrial network structure to the demands of stimulus-secretion coupling in pancreatic beta cells.

\section{Declaration of interest}

The authors declare that there is no conflict of interest that could be perceived as prejudicing the impartiality of the research reported.
Published by Bioscientifica Ltd. 


\section{Funding}

This study was funded by the German Diabetes Foundation (260-02-10).

\section{Acknowledgements}

The authors are indebted to Mr David Beattie (freelance medical writer/ UK) for editorial assistance in preparing the manuscript for publication.

\section{References}

Anello M, Lupi R, Spampinato D, Piro S, Masini M, Boggi U, Del Prato S, Rabuazzo AM, Purrello F \& Marchetti P 2005 Functional and morphological alterations of mitochondria in pancreatic beta cells from type 2 diabetic patients. Diabetologia 48 282-289. (doi:10.1007/ s00125-004-1627-9)

Bereiter-Hahn J \& Voth M 1994 Dynamics of mitochondria in living cells: shape changes, dislocations, fusion, and fission of mitochondria. Microscopy Research and Technique 27 198-219. (doi:10.1002/ jemt.1070270303)

Chen H \& Chan DC 2005 Emerging functions of mammalian mitochondrial fusion and fission. Human Molecular Genetics $\mathbf{1 4}$ (supplement 2) R283-R289. (doi:10.1093/hmg/ddi270)

Chen H \& Chan DC 2009 Mitochondrial dynamics-fusion, fission, movement, and mitophagy_in neurodegenerative diseases. Human Molecular Genetics 18 R169-R176. (doi:10.1093/hmg/ddp326)

Civitarese AE \& Ravussin E 2008 Mitochondrial energetics and insulin resistance. Endocrinology 149 950-954. (doi:10.1210/en.2007-1444)

Deng S, Vatamaniuk M, Huang X, Doliba N, Lian MM, Frank A, Velidedeoglu E, Desai NM, Koeberlein B, Wolf B, et al. 2004 Structural and functional abnormalities in the islets isolated from type 2 diabetic subjects. Diabetes 53 624-632. (doi:10.2337/ diabetes.53.3.624)

Detmer SA \& Chan DC 2007 Functions and dysfunctions of mitochondrial dynamics. Nature Reviews. Molecular Cell Biology 8 870-879. (doi:10.1038/nrm2275)

Dlaskova A, Spacek T, Santorova J, Plecita-Hlavata L, Berkova Z, Saudek F, Lessard M, Bewersdorf J \& Jezek P 2010 4Pi microscopy reveals an impaired three-dimensional mitochondrial network of pancreatic islet beta-cells, an experimental model of type-2 diabetes. Biochimica et Biophysica Acta 1797 1327-1341. (doi:10.1016/j.bbabio.2010.02.003)

Frieden M, James D, Castelbou C, Danckaert A, Martinou JC \& Demaurex N $2004 \mathrm{Ca}(2+)$ homeostasis during mitochondrial fragmentation and perinuclear clustering induced by hFis1. Journal of Biological Chemistry 279 22704-22714. (doi:10.1074/jbc.M312366200)

Hohmeier HE, Mulder H, Chen G, Henkel-Rieger R, Prentki M \& Newgard CB 2000 Isolation of INS-1-derived cell lines with robust ATP-sensitive $\mathrm{K}+$ channel-dependent and -independent glucosestimulated insulin secretion. Diabetes 49 424-430. (doi:10.2337/ diabetes.49.3.424)

Huang P 2006 Mitochondrial clustering induced by overexpression of the mitochondrial fusion protein Mfn2 causes mitochondrial dysfunction and cell death. European Journal of Cell Biology 86 289-302. (doi:10.1016/j.ejcb.2007.04.002)

Huang P, Galloway CA \& Yoon Y 2011 Control of mitochondrial morphology through differential interactions of mitochondrial fusion and fission proteins. PLOS ONE 6 e20655.

Hyde BB, Twig G \& Shirihai OS 2010 Organellar vs cellular control of mitochondrial dynamics. Seminars in Cell \& Developmental Biology 21 575-581. (doi:10.1016/j.semcdb.2010.01.003)

Ishihara N 2011 Molecular mechanism and physiological roles of mitochondrial fusion and fission. Seikagaku 83 365-373.
James DI, Parone PA, Mattenberger Y \& Martinou JC 2003 hFis1, a novel component of the mammalian mitochondrial fission machinery. Journal of Biological Chemistry 278 36373-36379. (doi:10.1074/jbc. M303758200)

Kuznetsov AV, Hermann M, Troppmair J, Margreiter R \& Hengster P 2010 Complex patterns of mitochondrial dynamics in human pancreatic cells revealed by fluorescent confocal imaging. Journal of Cellular and Molecular Medicine 14 417-425. (doi:10.1111/j.15824934.2009.00750.x)

Lee S, Jeong SY, Lim WC, Kim S, Park YY, Sun X, Youle RJ \& Cho H 2007 Mitochondrial fission and fusion mediators, hFis1 and OPA1, modulate cellular senescence. Journal of Biological Chemistry 282 22977-22983.

Liesa M, Palacin M \& Zorzano A 2009 Mitochondrial dynamics in mammalian health and disease. Physiological Reviews 89 799-845. (doi:10.1152/physrev.00030.2008)

Liu X \& Hajnoczky G 2011 Altered fusion dynamics underlie unique morphological changes in mitochondria during hypoxiareoxygenation stress. Cell Death and Differentiation 18 1561-1572. (doi:10.1038/cdd.2011.13)

Loson OC, Song Z, Chen H \& Chan DC 2013 Fis1, Mff, MiD49, and MiD51 mediate Drp1 recruitment in mitochondrial fission. Molecular Biology of the Cell 24 659-667. (doi:10.1091/mbc.E12-10-0721)

Lowell BB \& Shulman GI 2005 Mitochondrial dysfunction and type 2 diabetes. Science 307 384-387. (doi:10.1126/science.1104343)

Lu B 2009 Mitochondrial dynamics and neurodegeneration. Current Neurology and Neuroscience Reports 9 212-219. (doi:10.1007/s11910009-0032-7)

Maechler P \& Wollheim CB 2000 Mitochondrial signals in glucosestimulated insulin secretion in the beta cell. Journal of Physiology 529 49-56. (doi:10.1111/j.1469-7793.2000.00049.x)

Malmgren S, Nicholls DG, Taneera J, Bacos K, Koeck T, Tamaddon A, Wibom R, Groop L, Ling C, Mulder H, et al. 2009 Tight coupling between glucose and mitochondrial metabolism in clonal beta-cells is required for robust insulin secretion. Journal of Biological Chemistry 284 32395-32404. (doi:10.1074/jbc.M109.026708)

Molina AJ, Wikstrom JD, Stiles L, Las G, Mohamed H, Elorza A, Walzer G, Twig G, Katz S, Corkey BE, et al. 2009 Mitochondrial networking protects beta-cells from nutrient-induced apoptosis. Diabetes $\mathbf{5 8}$ 2303-2315. (doi:10.2337/db07-1781)

Mouli PK, Twig G \& Shirihai OS 2009 Frequency and selectivity of mitochondrial fusion are key to its quality maintenance function. Biophysical Journal 96 3509-3518. (doi:10.1016/j.bpj.2008.12.3959)

Otera H, Wang C, Cleland MM, Setoguchi K, Yokota S, Youle RJ \& Mihara K $2010 \mathrm{Mff}$ is an essential factor for mitochondrial recruitment of Drp1 during mitochondrial fission in mammalian cells. Journal of Cell Biology 191 1141-1158. (doi:10.1083/ jcb.201007152)

Palmer CS, Osellame LD, Stojanovski D \& Ryan MT 2011 The regulation of mitochondrial morphology: intricate mechanisms and dynamic machinery. Cellular Signalling 23 1534-1545. (doi:10.1016/j. cellsig.2011.05.021)

Palmer CS, Elgass KD, Parton RG, Osellame LD, Stojanovski D \& Ryan MT 2013 Adaptor proteins MiD49 and MiD51 can act independently of Mff and Fis1 in Drp1 recruitment and are specific for mitochondrial fission. Journal of Biological Chemistry 288 27584-27593. (doi:10.1074/ jbc.M113.479873)

Park KS, Wiederkehr A, Kirkpatrick C, Mattenberger Y, Martinou JC, Marchetti P, Demaurex N \& Wollheim CB 2008 Selective actions of mitochondrial fission/fusion genes on metabolism-secretion coupling in insulin-releasing cells. Journal of Biological Chemistry 283 33347-33356. (doi:10.1074/jbc.M806251200)

Santel A \& Fuller MT 2001 Control of mitochondrial morphology by a human mitofusin. Journal of Cell Science 114 867-874.

Schmitt H, Lenzen S \& Baltrusch S 2011 Glucokinase mediates coupling of glycolysis to mitochondrial metabolism but not to beta cell http://joe.endocrinology-journals.org DOI: 10.1530/JOE-16-0111
(C) 2016 Society for Endocrinology Printed in Great Britain
Published by Bioscientifica Ltd. 
damage at high glucose exposure levels. Diabetologia 54 1744-1755. (doi:10.1007/s00125-011-2133-5)

Serasinghe MN \& Yoon Y 2008 The mitochondrial outer membrane protein hFis1 regulates mitochondrial morphology and fission through self-interaction. Experimental Cell Research 314 3494-3507. (doi:10.1016/j.yexcr.2008.09.009)

Smirnova E, Griparic L, Shurland DL \& van der Bliek AM 2001 Dynaminrelated protein Drp1 is required for mitochondrial division in mammalian cells. Molecular Biology of the Cell 12 2245-2256. (doi:10.1091/mbc.12.8.2245)

Stiles L \& Shirihai OS 2012 Mitochondrial dynamics and morphology in beta-cells. Best Practice \& Research. Clinical Endocrinology \& Metabolism 26 725-738.

Stojanovski D, Koutsopoulos OS, Okamoto K \& Ryan MT 2004 Levels of human Fis1 at the mitochondrial outer membrane regulate mitochondrial morphology. Journal of Cell Science 117 1201-1210. (doi:10.1242/jcs.01058)

Twig G, Elorza A, Molina AJ, Mohamed H, Wikstrom JD, Walzer G, Stiles L, Haigh SE, Katz S, Las G, et al. 2008a Fission and selective fusion govern mitochondrial segregation and elimination by autophagy. EMBO Journal 27 433-446. (doi:10.1038/sj.emboj.7601963)

Twig G, Hyde B \& Shirihai OS $2008 b$ Mitochondrial fusion, fission and autophagy as a quality control axis: the bioenergetic view. Biochimica et Biophysica Acta 1777 1092-1097. (doi:10.1016/j. bbabio.2008.05.001)

Van Laar VS \& Berman SB 2009 Mitochondrial dynamics in Parkinson's disease. Experimental Neurology 218 247-256. (doi:10.1016/j. expneurol.2009.03.019)

Wells RC, Picton LK, Williams SC, Tan FJ \& Hill RB 2007 Direct binding of the dynamin-like GTPase, Dnm1, to mitochondrial dynamics protein Fis1 is negatively regulated by the Fis1 N-terminal arm. Journal of Biological Chemistry 282 33769-33775. (doi:10.1074/jbc. M700807200)

Westermann B 2008 Molecular machinery of mitochondrial fusion and fission. Journal of Biological Chemistry 283 13501-13505. (doi:10.1074/ jbc.R800011200)

Wollheim CB 2000 Beta-cell mitochondria in the regulation of insulin secretion: a new culprit in type II diabetes. Diabetologia 43 265-277. (doi:10.1007/s001250050044)

Yoon Y, Krueger EW, Oswald BJ \& McNiven MA 2003 The mitochondrial protein hFis1 regulates mitochondrial fission in mammalian cells through an interaction with the dynamin-like protein DLP1. Molecular and Cellular Biology 23 5409-5420. (doi:10.1128/ MCB.23.15.5409-5420.2003)

Yoon Y, Galloway CA, Jhun BS \& Yu T 2011 Mitochondrial dynamics in diabetes. Antioxidants \& Redox Signaling 14 439-457.

Yu T, Fox RJ, Burwell LS \& Yoon Y 2005 Regulation of mitochondrial fission and apoptosis by the mitochondrial outer membrane protein hFis1. Journal of Cell Science 118 4141-4151. (doi:10.1242/jcs.02537)

Zhang Y \& Chan DC 2007 Structural basis for recruitment of mitochondrial fission complexes by Fis1. PNAS 104 18526-18530. (doi:10.1073/pnas.0706441104)

Zhu PP, Patterson A, Stadler J, Seeburg DP, Sheng M \& Blackstone C 2004 Intra- and intermolecular domain interactions of the C-terminal GTPase effector domain of the multimeric dynamin-like GTPase Drp1. Journal of Biological Chemistry 279 35967-35974. (doi:10.1074/jbc.M404105200)

Zorzano A, Liesa M \& Palacin M 2009 Mitochondrial dynamics as a bridge between mitochondrial dysfunction and insulin resistance. Archives of Physiology and Biochemistry 115 1-12. (doi:10.1080/13813450802676335)

Received in final form 26 April 2016

Accepted 13 May 2016

Accepted Preprint published online 13 May 2016
(C) 2016 Society for Endocrinology Printed in Great Britain
Published by Bioscientifica Ltd. 